\title{
A Mathematical Model for Identifying Truth in Observations Made within Individual Human Self-Awareness
}

Andy E. Williams, Nobeah Foundation, Nairobi, Kenya

\begin{abstract}
A large body of work purports to identify the functions of the human system through observations that can be validated within human self-awareness. That is, using observations that can be made in the human self-awareness to validate what in this paper are called human-centric models of the functionality of the human system, or human-centric functional models. This body of work, accumulated over thousands of years, has been largely inaccessible to the scientific study of consciousness and other functions because it's written in terms that require a very different training, which is training in observing one's self-awareness. Making scientific or mathematical use of observations made within human self-awareness is a challenge. What can be observed to be true in one's self-awareness, and what can be proved true mathematically, have so far been two different things. Representing an experiment as a channel through which information representing truth can be transmitted, this paper explores the use of Information Theory to gauge the capacity of an experiment in self-awareness to identify a truth, such as determining the validity of any given function defined by a functional model for consciousness. In providing this mechanism to prove that an awareness exists and that it reflects truth, this paper attempts to make the entire set of observations made using human selfawareness accessible to mathematicians and scientists.
\end{abstract}

\section{Introduction}

Since some functionality of consciousness or cognition can't yet be measured externally, experiments to confirm theories about that functionality must involve the subject's observations of their own conscious self-awareness. In order for functionality of consciousness or cognition to be observed in the subject's self awareness, that functionality must be defined in human-centric terms. That is, the units of functionality must be defined in terms that enable a human being to detect that functionality within their innate human self-awareness, rather than requiring them to accept the validity of any theoretical framework. This approach to defining models of the functionality of the human system is referred to here as human-centric functional modeling.

\section{Definition of Truth of Awareness in Mathematical Terms}

In order to enable the mathematics and scientific community to utilize experiments requiring observations in the human self-awareness, this paper attempts to apply information theory to the problem of proving that an awareness exists. In doing so it attempts to provide these disciplines with access to the entire set of observations made using functional models of the human system that can be validated within human self-awareness. Providing this whole set of ideas to be reapplied in mathematics, creates promising opportunities for breakthroughs, which can happen when existing ideas are generalized so they can be reused in new domains where they apply.

Consider the truth of an observation that can be made within an individual's self awareness to be a signal. In the Functional Modeling Framework (FMF) [1] awarenesses are output (transmitted by) awareness processes of the consciousness. Therefore, according to information theory, the ability of any truth to be transmitted depends on characteristics of these awareness processes.

In particular, information theory [2] gives the capacity of a channel to transmit information as: 


\section{$C=\max _{f} I(X ; Y)$}

This capacity has the property related to communicating at information rate $\mathrm{R}$ (where $\mathrm{R}$ is usually bits per symbol), that any for information rate $\mathrm{R}<\mathrm{C}$ and coding error $\varepsilon>0$, for large enough $\mathrm{N}$, there exists a code of length $\mathrm{N}$ and rate $\geq \mathrm{R}$ and a decoding algorithm, such that the maximal probability of block error is $\leq \varepsilon$; that is, it is always possible to transmit with arbitrarily small block error. In addition, for any rate $\mathrm{R}>\mathrm{C}$, it is impossible to transmit with arbitrarily small block error.

\section{Defining the Channel Capacity of Awareness Processes}

The truth of an assertion can be observed though an individual's processes of self-awareness if they have processes with the capacity for the required awareness, and if they can execute those processes reliably. In intuitive terms, a blind person is not a reliable observer of awarenesses of visual stimuli. Or a superstitious person is not a reliable observer of awarenesses they are superstitious about. An assertion is proposed here to be true within the individual's self-awareness if the collective capacity of all available awareness processes transmits that proposed truth with an error that is small compared to the information in the observation.

As an intuitive example, an individual believes they saw a ghost. Is that observation true within their self-awareness? Are they lying? Or are they being honest but are just a poor observer of their selfawareness because of superstitions resulting in a great many inconsistencies in their observations (i.e. resulting in a high noise ratio in their observations). If awarenesses aren't repeatable, if awarenesses contradict each other, these all define either a poor observation or a poor observer.

\section{Representing Awareness Processes so Channel Capacity can be Determined}

The FMF defines processes to consist of a set of functions, each of which function has a set of inputs, a set of outputs, and additional information outside of the inputs and outputs that determines the execution of each function. In the FMF the consciousness is represented as executing a library of awareness processes. These processes take awarenesses as input, and produce awarenesses as output. In other words, since awarenesses in the FMF are defined as points in an awareness space, these processes map from one point in the awareness space (one awareness) to another.

Unfortunately the awareness space has not been precisely defined. And therefore the awareness processes can't be precisely defined, so the above equation can't yet be evaluated to asses truth in an individual's self-awareness. However, qualitatively the idea is still quite powerful as it enables a functional definition of truth to be elaborated in terms of repeatability and reliability of an observation.

\section{Designing Experiments in Human Self-Awareness}

Since some functionality of consciousness and cognition can't yet be externally measured, testing theories of consciousness or cognition which involve that functionality must be done with experiments that rely on the individual subject's observation of their own self-awareness.

In terms of information theory, truth is the signal from the consciousness process that we are trying to detect, and falsehood is noise in that signal. If we want to determine whether a pop or a hiss on a recorded music track is part of the song (signal), or is noise, we will compare it with recordings made 
from different master copies so that the same error is not systematically propagated. We will also make the comparison using different playback equipment.

In the same way, if we want to determine whether an awareness is true, we can have multiple people try the same experiment. And we can design different experiments that seek to validate the same awareness.

\section{Implications}

A large body of work has been done over thousands of years to define human-centric functional models of the human system. These models are extremely useful in the study of consciousness because they define the problem of consciousness experientially. Since these traditions provide experientially verifiable definitions of terms that when defined intellectually are ambiguous, this in itself is a tremendous contribution to research in consciousness or cognition. In other words, intellectual reasoning has a capacity to arrive at truth that is finite (limited to cases in which adequate reasoning and the facts to plug into that reasoning exist) and potentially unreliable (reliable only where such reasoning is simple enough to be accurately computed). Experiential reasoning has a capacity to arrive at truth that is infinite (the truth of an infinite number of observations can be experienced) and reliable (experience can reliably be observed wherever an observation can be accurately identified as one's experience). The more experiential and less intellectual the discussion of cognition, potentially the more capable that discussion is of reliably converging on the truth.

Indeed, even in creating mathematical models of consciousness it is important to clarify the problem being solved not only from a mathematical perspective, but also from the perspective of this humancentric functional modeling. For example, this human-centric approach requires being able to validate the difference between the function of conscious well-being (as opposed to the function of physical, emotional, or cognitive well-being) in simple experiments that can be performed in one's own selfawareness.

Another example of the need to remove ambiguity is the suggestion that consciousness processes "experience" as input. That is just one of the areas where such a human-centric functional perspective might add tremendous clarity. In the human-centric functional model, experience can consist of a combination of both awarenesses and concepts. Experience can contain a set of awarenessess, which are the input to and output from the consciousness process. And it can contain a set of concepts, which are input to and output from the cognition process (the mind). Without a human-centric functional model to clarify this distinction, and to confine the behavior of the consciousness to the awareness space, as well to confine the behavior of the cognitive system to the conceptual space so this ambiguity cannot be reintroduced, the term "experience" may be too ambiguous to permit research to converge on a testable conclusion. Another example, are discussions on "pure awareness" among consciousness researchers. These discussions may confuse awareness (the only thing that consciousness processes in this functional model), with other concepts. As an example, for some, pure awareness may mean "heightened" or "expanded" awareness. In the human-centric functional modeling perspective, since the input to and output from the consciousness process are awarenesses, "pure awareness" just means "lack of thinking". The experience of pure awareness simply means recognizing the difference between the function of consciousness (awareness), and the function of cognition (thinking), and then simply refraining from the latter. Expanding one's awareness is an entirely different thing, but that expansion is also well-defined within a human-centric modeling framework.

There are critical differences and relative benefits between human-centric functional models that can potentially be validated experientially within human self-awareness, and abstract mathematical models 
that can be useful in deriving very complex relationships. Models that can be validated within the selfawareness can reliably be tested experimentally with awareness of any entity in this existence that human beings may or may not yet have encountered. Abstract mathematical models can be used to extrapolate relationships extremely precisely to make new predictions, even where those relationships can't be tested within human self-awareness.

As an example in the case of human-centric functional models, when exposed to psychoactive substances never before tested on humans, a human-being can simply observe their awareness of the impact of those substances. No functionality within the human sensory system, or within the human system of cognition, can be outside the capacity of the human awareness of those systems.

As an example in the case of mathematical models, the Integrated Information Theory (IIT) model of consciousness [3] uses a chain of reasoning to define a precise mathematical quantity phi that can't be validated within human self-awareness. Any of the steps in this chain of reasoning might prove to be either correct or incorrect. If incorrect, then the final conclusion might also be off. As a result this method is prone to errors in the reasoning path. However it also enables probing the properties of consciousness at far greater resolution than self-awareness is capable of.

These two fundamentally different approaches might be summarized as intellectual reasoning and natural (experiential) logic. An analogy to intellectual reasoning is navigation using a set of instructions in terms of number of steps, directions to turn right or left, and street names. These directions might be extremely accurate, but if there is any error or gap in the instructions the individual will be lost and have to retrace their steps to a known point. And analogy to natural logic that can be confirmed within the individual's self-awareness is like navigation by the stars. The individual might not even notice the street names. And as long as the star is visible, it can be used to navigate this entire existence. Both approaches can complement each other.

\section{Conclusions}

There are many experiments that can be defined in which observations are made within individual human self-awareness. For many of the functions of this human system, including consciousness, no external mechanism yet exists to measure those functions, so observation within human self-awareness is the only means possible to validate the truth of an assertion. This paper suggests that truth in such observations can be identified in part by assessing the repeatability of the observation, and by assessing the reliability of the observer.

Breakthroughs happen when existing ideas are generalized so they can be reused in new domains where they apply. The human-centric functional modeling approach provides a whole set of ideas to be generalized and reapplied in mathematics and the sciences. If correct, this model for identifying truth in those observations enables that reuse to occur.

\section{References}

[1] Williams, A. E. (2020, April 16). A Human-Centric Functional Modeling Framework for Defining and Comparing Models of Consciousness and Cognition. https://doi.org/10.31234/osf.io/94gw3 [2] S. Verdu and Te Sun Han, "A general formula for channel capacity," in IEEE Transactions on Information Theory, vol. 40, no. 4, pp. 1147-1157, July 1994.

[3] Tononi, G. The integrated information theory of consciousness: an updated account. Arch. Ital. Biol. $150,56-90(2012)$. 